\title{
Heat production in a feeding matrix formed on carrion by communally breeding beetles
}

\author{
Szymon Matuszewski ${ }^{12^{*}}$ (D) and Anna Mądra-Bielewicz ${ }^{1,2}$
}

\begin{abstract}
Insects regulate their body temperature mostly behaviourally, by changing posture or microhabitat. Usually they use heat that is already present in the environment. Sometimes, however, they may manipulate the environment to affect, focus or benefit from thermogenesis. Carrion beetles create a feeding matrix by applying to cadaver surface anal or oral exudates. We tested the hypothesis that the matrix, which is formed on carrion by communally breeding beetle Necrodes littoralis L. (Silphidae), produces heat that enhances insect fitness. Using thermal imaging we demonstrate that heat produced in the matrix formed on meat by adult or larval beetles is larger than in meat decomposing without insects. Larval beetles regularly warmed up in the matrix. Moreover, by comparing matrix temperature and larval fitness in colonies with and without preparation of meat by adult beetles, we provide evidence that formation of the matrix by adult beetles has deferred thermal effects for larval microhabitat. We found an increase in heat production of the matrix and a decrease in development time and mortality of larvae after adult beetles applied their exudates on meat in the pre-larval phase. Our findings indicate that spreading of exudates over carrion by Necrodes larvae, apart from other likely functions (e.g. digesting carrion or promoting growth of beneficial microbes), facilitates thermoregulation. In case of adult beetles, this behaviour brings distinct thermal benefits for their offspring and therefore may be viewed as a new form of indirect parental care with an important thermal component.
\end{abstract}

Keywords: Insect thermoregulation, Animal behaviour, Parental care, Carrion ecology, Defecation

Temperature is a key component of animal environment. Many species, particularly insects, are more or less dependent on external heat $[1,2]$. Animals frequently use heat that is already present in the environment, through changing body orientation (e.g. basking), selecting thermally attractive microhabitat or optimising its thermal characteristics (e.g. aggregating) [3-5]. Rarely, however, they transform the environment to affect, focus or benefit from thermogenesis. Some Indo-Pacific

\footnotetext{
* Correspondence: szymmat@amu.edu.pl

'Laboratory of Criminalistics, Adam Mickiewicz University, Święty Marcin 90, 61-809 Poznań, Poland

${ }^{2}$ Wielkopolska Centre for Advanced Technologies, Adam Mickiewicz University, Uniwersytetu Poznańskiego 10, 61-614 Poznań, Poland
}

megapode birds, e.g. Australian brush-turkey (Alectura lathami) construct their incubation mounds from plant litter, where the composting generates heat that is used for egg hatching $[6,7]$. Some crocodilians make similar incubation mounds, in which heat is produced by decomposing plant material $[6,8,9]$. A different example is the behaviour of tent caterpillars that build silk tents where they aggregate to take advantage of metabolic thermogenesis [10]. Habitat transformation to facilitate thermogenesis may occur also in carrion insects. Blow flies (Calliphoridae) or carrion beetles (Silphidae) use cadavers mostly for breeding and their larvae are main carrion reducers in some terrestrial environments [11-13]. Necrophagous larvae usually feed in aggregations [14], 
which may have much higher inner temperature than ambient air (by $10-30{ }^{\circ} \mathrm{C}$ ). This effect, called the maggot-mass effect, was originally discovered in blow flies [5, 15-19], but has also been reported for Necrodes beetles (Silphidae) [20]. The inner heat of these aggregations was hypothesized to derive from microbial activity [21, 22], larval exothermic digestive processes [18] or larval frenetic movements [19, 23]. However, there is no direct evidence to support any of these mechanisms.

Carrion beetles form a feeding matrix on cadavers (also called "a biofilm-like matrix") by spreading over its surface anal and oral exudates [24]. Carrion smearing was originally described in adult burying beetles (Silphidae: Nicrophorus) [25]. This behaviour was hypothesized to moisturize carrion [25], facilitate digestion [25-27], suppress microbial competitors [24, 28-31], deter insect competitors by reducing carrion-originating attractants $[25,32,33]$, support larval aggregation [25] or development [28] or seed mutualistic microbes and transmit them to offspring [26, 34-36]. Exudates of adult or larval burying beetles were found to contain antimicrobial compounds [37-41]. Moreover, presumptively mutualistic microbes (e.g. Yarrowia yeasts) were abundantly identified in carrion beetle guts and the matrix formed on carrion by the beetles [24, 26, 36, 42, 43]. These findings indicate that feeding matrix on carrion is a complex microenvironment emerging from interactions between burying beetles, microbes and a putrefying resource. Although cadaver smearing behaviour has not been reported in other carrion beetles, there is indirect evidence suggesting that formation of the matrix is more prevalent among these beetles [37, 42]. The matrix probably brings several benefits for the beetles and their scope may vary between the species.

Carrion beetles (Silphidae) are divided into Nicrophorinae (burying beetles), and Silphinae [44]. The latter subfamily is more diverse, with necrophagous, predatory and phytophagous species [45, 46]. Necrodes beetles are members of Silphinae, grouped with Ptomaphila, Oxelytrum and Diamesus at the base of the subfamily [46-48]. Necrodes colonizes large vertebrate cadavers, where its larvae feed on carrion tissues and under favourable conditions may reduce them into dry remains $[49,50]$. Beetles start visiting carrion after it becomes bloated (under summer temperatures usually $4-8$ days after death) and after some time many of them (even hundreds) may be present on a cadaver $[49,51-53]$. Females lay eggs (3050 per female) in a nearby soil and larvae abundantly colonize carrion during late decomposition $[49,51,52]$. Adult beetles are usually absent when larvae colonize the resource. In Central European forests during the summer, adult $N$. littoralis were present 3-8 days on pig carcasses and usually 5-7 days elapsed between the arrival of the first adult beetles and the first larvae [54].
Under laboratory conditions, when food and temperature are optimal, adult beetles oviposit within 12 days of provisioning them with fresh meat. The egg stage of $N$. littoralis lasts on average 3.4 days at $22^{\circ} \mathrm{C}$ and 4.9 days at $18{ }^{\circ} \mathrm{C}$ (Gruszka, personal communication). Necrodes larvae feed in aggregations, which form in the warmest place and relocate in response to changes in the heat source location [14]. This indicates that heat plays an important role in the formation and maintenance of larval aggregations in Necrodes [14]. After larvae stop feeding, they pupate in a nearby soil [49]. When disturbed, adult Necrodes beetles spray defensive anal secretions (frequently mixed with excretions), which have a strong repellent effect against other insects $[55,56]$ and a significant antibacterial action [37]. However, its addition directly to the carrion has not been reported. Necrodes beetles, in contrast to burying beetles, colonize large carrion, breed there communally and are considered as species without parental care $[45,46,51]$.

We found that Necrodes littoralis L. under laboratory conditions forms on meat a feeding matrix, similarly to burying beetles. Because larvae of this species are distinctly heat-oriented, and external heat certainly enhances their fitness, we formulated a hypothesis that the feeding matrix produces heat, which is beneficial for the larvae. Because both adult and larval beetles were found to spread their exudates over meat forming the matrix, we tested also whether the application of exudates by adult beetles affects heat production of the matrix during the larval feeding phase and eventually brings thermal benefits for the larvae.

\section{Results}

\section{Heat emission in feeding matrix formed on carrion by Necrodes beetles}

To test if feeding matrix produced by $N$. littoralis generates heat, we monitored with thermal imaging conditions in colonies of adult (A) and larval (L) beetles subsequently feeding on meat $(M)$ (hereafter $M / A+L$ setup, adult beetles were removed before adding the larvae) and in colonies of larval beetles only (hereafter $\mathrm{M}+$ $\mathrm{L}$ setup), using as a reference the equivalent meat setup without the insects (hereafter M setup, Fig. 1). We found that adult and larval beetles spread their exudates over meat to form a greasy feeding matrix that covers the meat and surrounding soil, the surface of which was enlarging with colony age (Fig. 2). The matrix had a higher temperature than the background (Fig. 3) and beetle larvae were regularly warming up on its surface (Fig. 4).

To investigate the effect of environmental temperature on heat emission in a matrix, we compared larval colonies $(\mathrm{M}+\mathrm{L}$ setup) reared under different temperatures, using as a reference paired containers with meat only ( $\mathrm{M}$ setup). The comparison revealed that the heat 


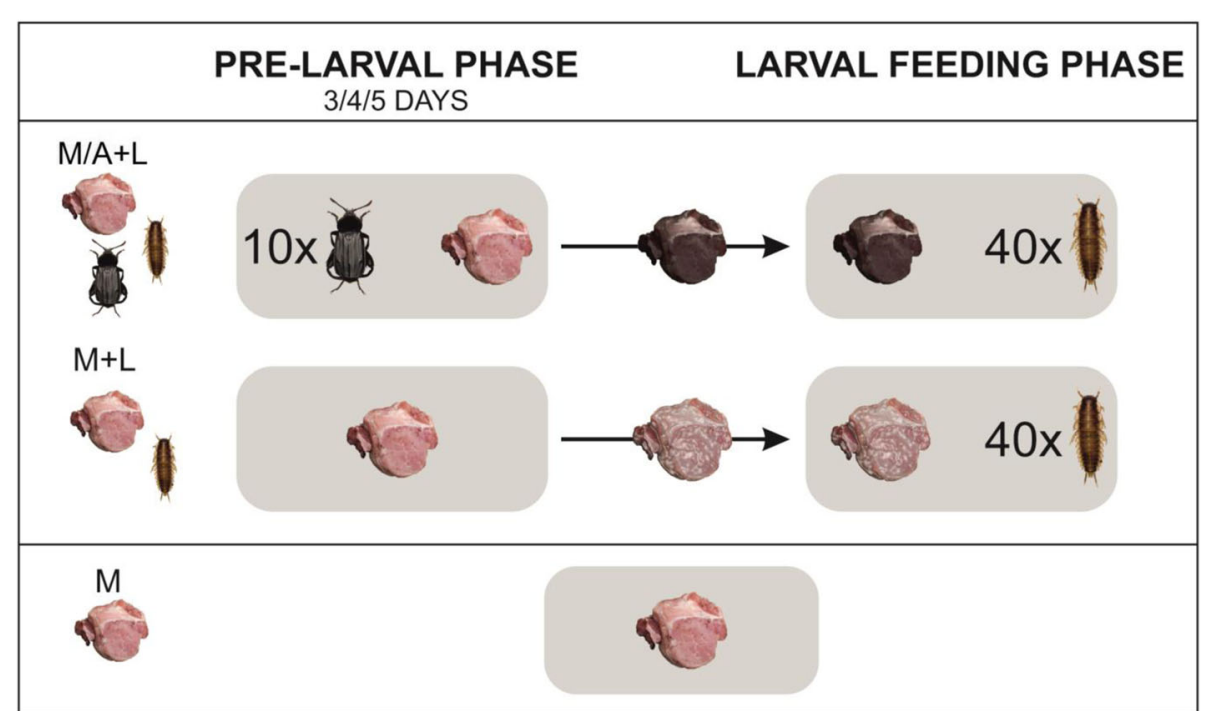

Fig. 1 Experimental design to study the heat production of a feeding matrix formed on meat by carrion beetle Necrodes littoralis and the development of its larvae in colonies with and without adult beetles presence in the pre-larval phase (respectively $M / A+L$ and $M+L$ setups). Trials with meat only ( $\mathrm{M}$ setup) were used as the reference

production in the matrix formed by larvae increased with rearing temperature $\left(F_{3,35}=59.4, N=39, P<0.001\right.$, Fig. Ia, b in supplementary material) and was significantly larger than in meat alone at all rearing temperatures, apart from $16^{\circ} \mathrm{C}\left(F_{1,35}=87.5, N=39, P<0.001\right.$, Fig. Ib in supplementary material).

By quantifying the average temperature of the matrixcovered surface $(\mathrm{M} / \mathrm{A}+\mathrm{L}$ and $\mathrm{M}+\mathrm{L}$ setups) and meat without the beetle-derived matrix ( $\mathrm{M}$ setup), we found that heat emission in the matrix was significantly larger than in meat alone $\left(F_{1,27}=122, N=30, P<0.001\right.$, Fig. 5a, b). The temperature of the matrix revealed a steady increase until meat resources were depleted and larvae stopped feeding (Fig. 5a). When adult beetles (M/A + L setup) applied their exudates in the pre-larval phase, the temperature of the matrix was significantly higher in the larval feeding phase, as compared to the colonies of larval beetles only $\left(F_{1,27}=154.8, N=30, P<0.001\right.$, Fig. 5 c). Moreover, heat production in the matrix during the larval feeding phase became larger, when we extended the pre-larval phase $\left(F_{2,27}=24, N=30, P<0.001\right.$, Fig. 5a, c).

\section{Feeding matrix, larval fitness and parental care in Necrodes beetles}

To test if spreading the exudates over meat by adult beetles affects the fitness of larvae, we compared

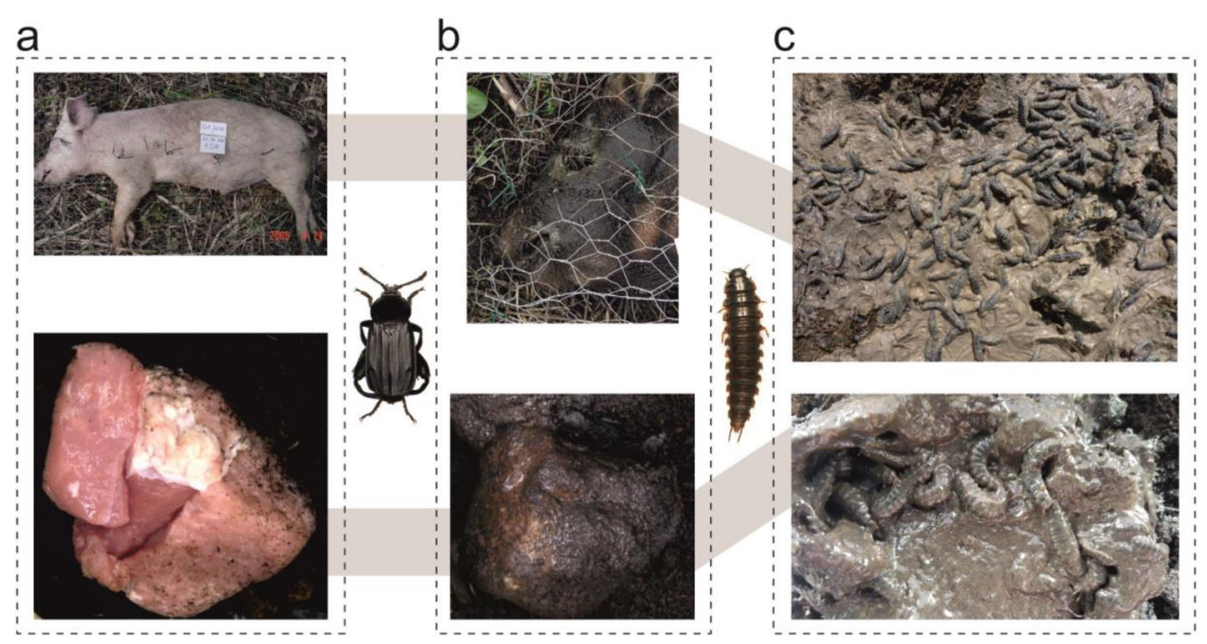

Fig. 2 The feeding matrix formed by adult or larval Necrodes littoralis on pig cadaver under field conditions (top panel) and on meat in the laboratory (bottom panel). $\mathbf{a}$ - fresh resources, $\mathbf{b}$ - the matrix formed by adult beetles, $\mathbf{c}$ - the matrix formed by larvae 

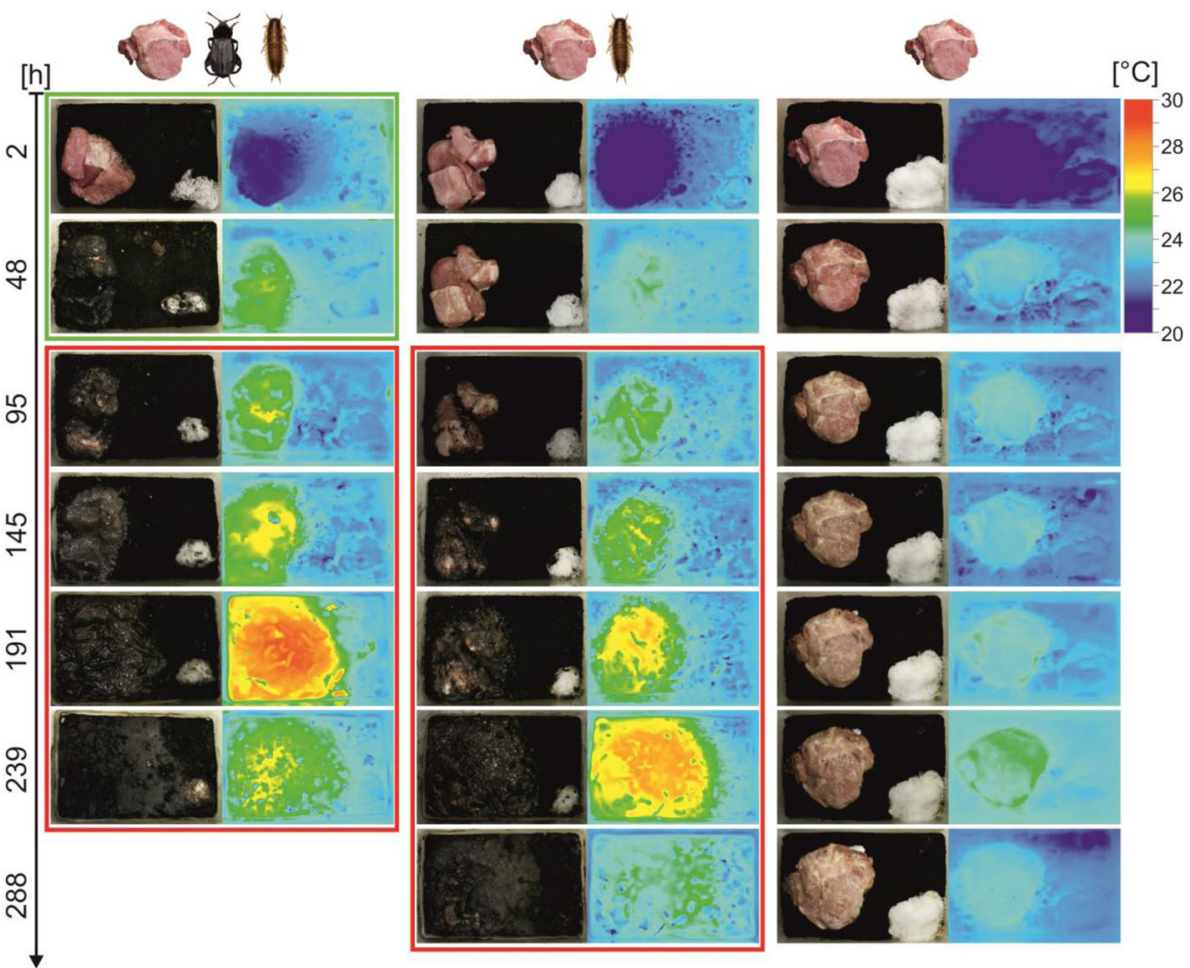

Fig. 3 Heat production of the feeding matrix in colonies with adult and larval Necrodes littoralis (left column), with larval beetles only (middle column) and without the beetles (right column). The green frame includes pictures of adult beetle colonies, the red frame pictures of larval beetle colonies. Pictures without a frame show meat decomposing without insects

development times of larvae and pupae, larval mortality and mass of postfeeding larvae between colonies with and without the presence of adult beetles in the prelarval phase $(\mathrm{M} / \mathrm{A}+\mathrm{L}$ and $\mathrm{M}+\mathrm{L}$ setups). Larvae developed significantly faster after adult beetles prepared the meat $\left(F_{1,27}=15, N=30, P<0.001\right.$, Fig. 6a), differences in the pupal development time were not significant $\left(F_{1,27}=\right.$ $0.8, N=30, P=0.39$, Fig. $6 \mathrm{~b})$. Mortality of larvae was significantly lower in colonies with meat prepared by adult beetles $\left(F_{1,27}=8.3, N=30, P<0.01\right)$; differences between the treatments were the biggest in the case of the 3-day pre-larval phase (Fig. 7a). An increase in the duration of the pre-larval phase resulted in higher larval mortality $\left(F_{1,27}=3.5, N=30, P=0.045\right.$, Fig. 7a). Application of exudates by adult beetles revealed no significant effect on the mass of postfeeding larvae $\left(F_{1,27}=2.4, N=30, P=\right.$ 0.13, Fig. 7b).

\section{Discussion}

We demonstrated that Necrodes beetles form a feeding matrix on carrion in which heat is produced and beetle larvae warm up while feeding. The heat emission recorded in insect colonies was highly consistent in space and time with the matrix formation (Fig. 3). Since there is no known endothermic mechanism in insect larvae except minimal metabolic heat generation $[2,5]$, we rejected a hypothesis that the larvae contributed endothermically to the heat increase. Even if some unknown mechanism operated in our colonies, they consisted of only 40 larvae, so the mechanism would probably be not effective in raising and maintaining the temperature over the entire matrix-covered surface. Moreover, our close-up thermal images (Fig. 4) demonstrated that the heat of the larvae was a function of the heat of the matrix and not vice versa. Therefore, we interpret our findings as evidence that the heat is not endothermically generated by the larvae, but is produced in the matrix, and that the larvae contribute to this only by forming and maintaining the matrix. Although there are reports that pig cadavers decomposing without insects may heat up due to microbial activity [22], we compared beetle colonies against meat only and the heat emission was always much larger with the beetles. Therefore, beetle-derived matrix on meat surface may be viewed as a single key factor responsible for the heating effects reported in this study. However, heating of the feeding matrix and the cadaver may superimpose and we suspect that in a natural, large cadaver situation this may lead to an increased total effect.

In the experiments, we used fresh pork meat (pieces of 80-150 g), whereas in the field situation N. littoralis colonizes large cadavers in a late decomposition stage, with 


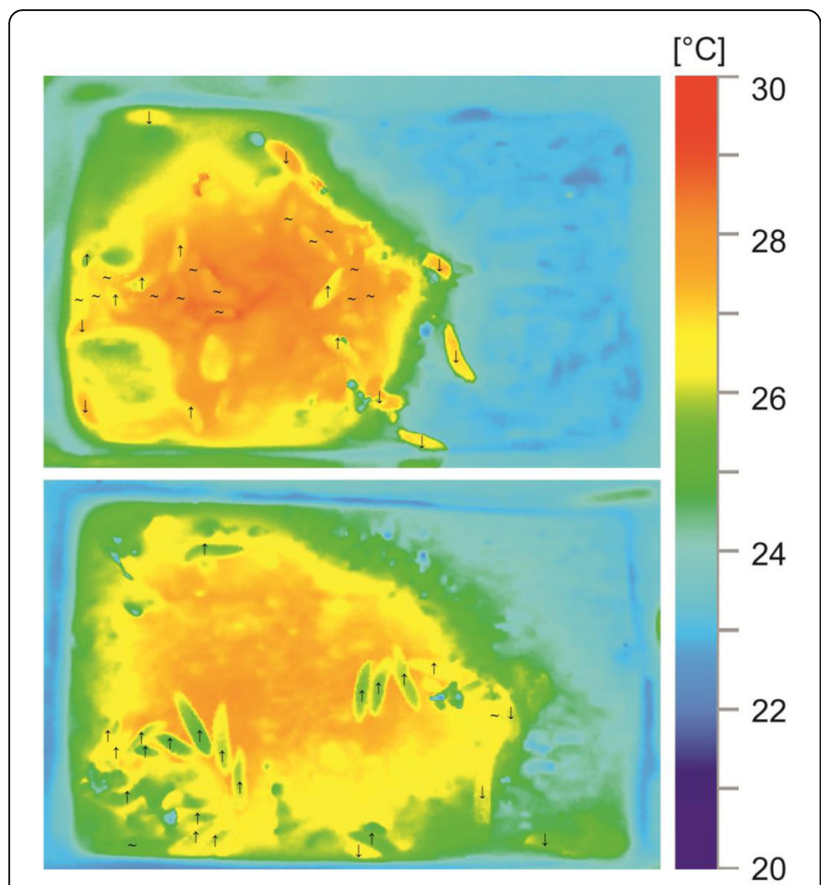

Fig. 4 Thermal images of larval Necrodes littoralis warming up in the feeding matrix. Images demonstrate that heat of the larvae is a function of the matrix heat. Lavae present on the matrix may be classified into: 'warming' ( $\uparrow$ ) and 'warmed' ( ). Temperature of the 'warming' larvae is much lower than temperature of the matrix in their vicinity and temperature of the 'warmed' larvae is the same or only slightly lower. There is a clear heat gradient from the outside to the inside in the 'warming' larvae, which indicates they are warming up in the matrix. There are also 'cooling' ( $\downarrow)$ larvae, with higher body temperature than in their vicinity and a heat gradient from the inside to the outside. However, they are present only outside of the matrix. Assuming that larvae contribute endothermically to the heat of the matrix, the 'cooling' larvae should be present on the surface covered with the matrix. As this is not the case, the images demonstrate that heat is not endothermically generated by the larvae, but is produced in the matrix. Moreover, larvae are warming up while staying on the matrix. Because larvae frequently move through the matrix and change their position in relation to the hot spots, they take advantage of the average heat of the matrix and usually they do not reach the maximum temperatures of the matrix regular occurrence on carcasses larger than $30 \mathrm{~kg}[14$, 57]. This may raise doubts as to the translation of our findings to the natural, field situation. When blow flies are absent (in central Europe during early spring), $N$. littoralis may drive active decay of large carrion, replacing blow fly larvae $[50,58]$. In such cases several hundreds of adult beetles may visit a cadaver and thousands of $N$. littoralis larvae may be present, with minimal or absent colonization by other necrophagous insects, which amounts to the carrion monopolization by these beetles (Fig. IIa, b in supplementary material). When a cadaver is infested with blow fly larvae and it is large enough not to be entirely consumed by blow flies, the active decay driven by maggots is frequently followed by the active decay driven by larval $N$. littoralis [50]. In such cases, $N$. littoralis may be also very abundant and monopolize carrion that is left after blow flies cease feeding (Fig. IIc, $\mathrm{d}$ in supplementary material). Therefore, the monopolization of carrion by $N$. littoralis occurs in a natural, field situation. Moreover, during our field experiments, we regularly observed that Necrodes larvae formed a feeding matrix on such monopolized carcasses and were able to spread their exudates over large carrion parts and even the entire surface of a large pig carcass (Fig. II in supplementary material). When larval aggregations of $N$. littoralis were present, they had distinctly higher inner temperature than ambient air [20]. Therefore, the formation of feeding matrix by $N$. littoralis and heat production in the matrix are not the laboratory artefacts, but natural phenomena. The mating and oviposition behaviour of adult beetles that were provided with fresh pieces of pork meat were normal; beetles eagerly undertook these actions in our colonies. Moreover, premature development of the $N$. littoralis beetles that were reared on the fresh pork diet to collect developmental data for forensic applications was also normal [59]. Although beetles in the field situation feed on a more

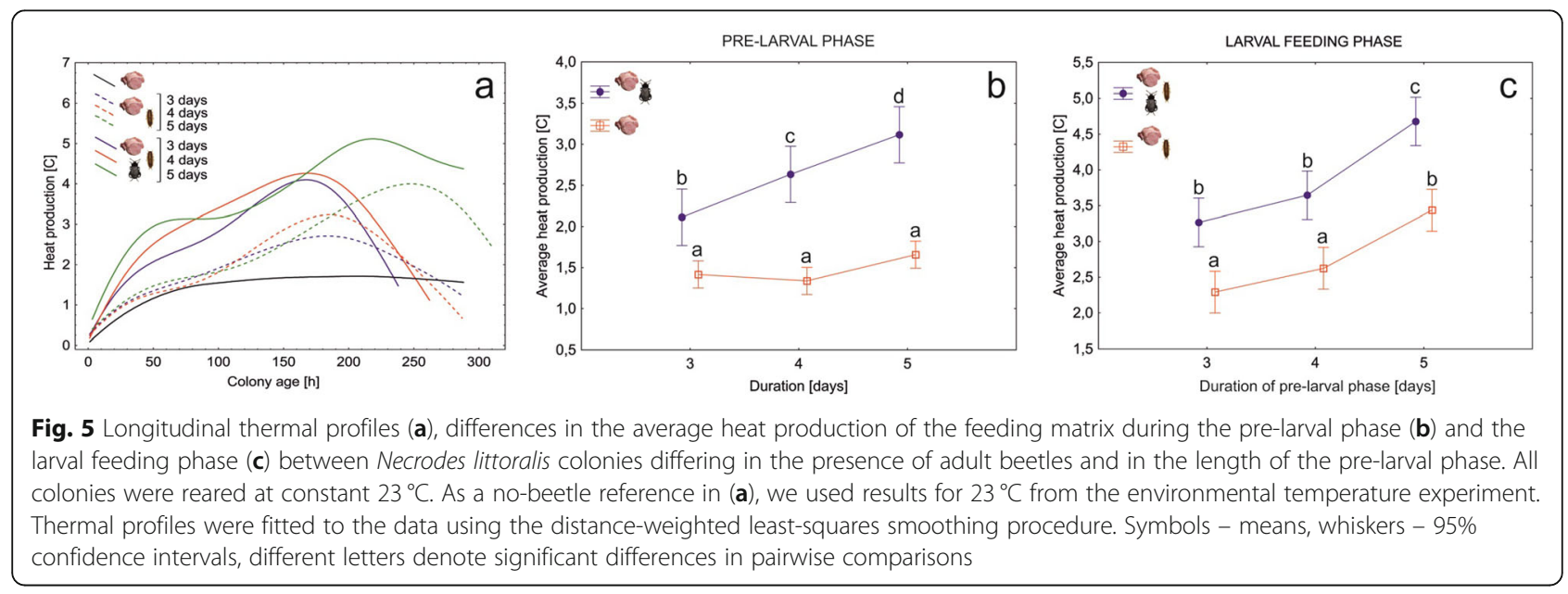



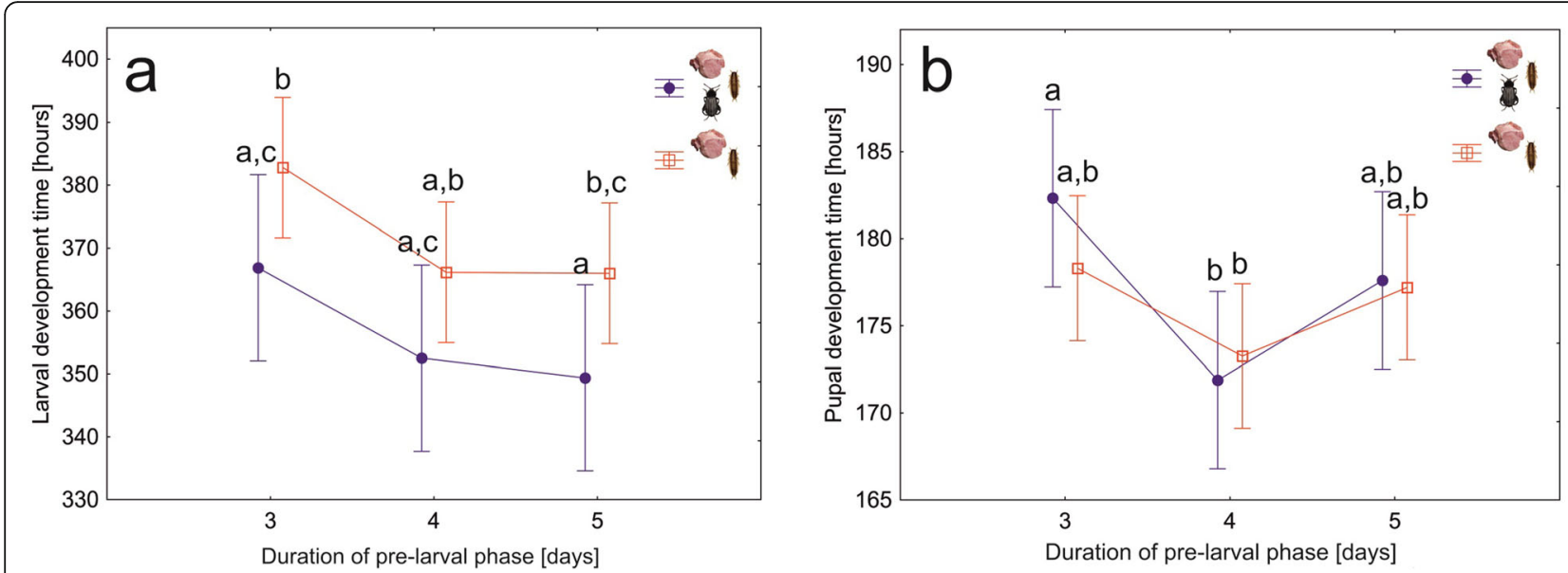

Fig. 6 Average larval (a) and pupal (b) development times for Necrodes littoralis colonies differing in the presence of adult beetles during the prelarval phase and in the duration of this phase. Larval development times include feeding (on meat) and postfeeding (in Petri dishes) phases. Symbols - means, whiskers - 95\% confidence intervals, different letters denote significant differences in pairwise comparisons

decomposed carrion, with a more diverse and abundant microbiome [60], using the fresh meat in our experiments cannot explain the differences that were observed between the treatments, as in all the setups fresh pork was used. Experimental design with intact carcasses (e.g. rabbits) would be more ecologically relevant, but also less controllable and more difficult to replicate in the laboratory.

The feeding matrix is a complex microenvironment. Its properties certainly change along the decomposition timeline, with a beetle life stage that participates in its formation or with initial condition of carrion. Beetles contribute to its formation and maintenance by spreading their exudates over carrion surface. The anal exudates of Necrodes adult beetles are mixtures of anal secretions with fecal material $[55,56]$, whereas exudates of larvae are probably dominated by feces. Beetles may also apply oral exudates. Exudates of adult and larval beetles may contain digestive compounds, antimicrobials, beneficial microbes, undigested food etc. Accordingly, beetles may contribute to the matrix formation and maintenance mostly through exodigestion of carrion and manipulation of its microbial content with probable large differences in this respect between species and life stages.

Although our results do not support any of the putative mechanisms for the heat generation in the matrix, two general hypotheses may be formulated in this respect. The heat production may involve the activity of microbes. Feeding matrix on carrion, thoroughly investigated in burying beetles, is a mixture of microbes, their metabolites and compounds released into the matrix
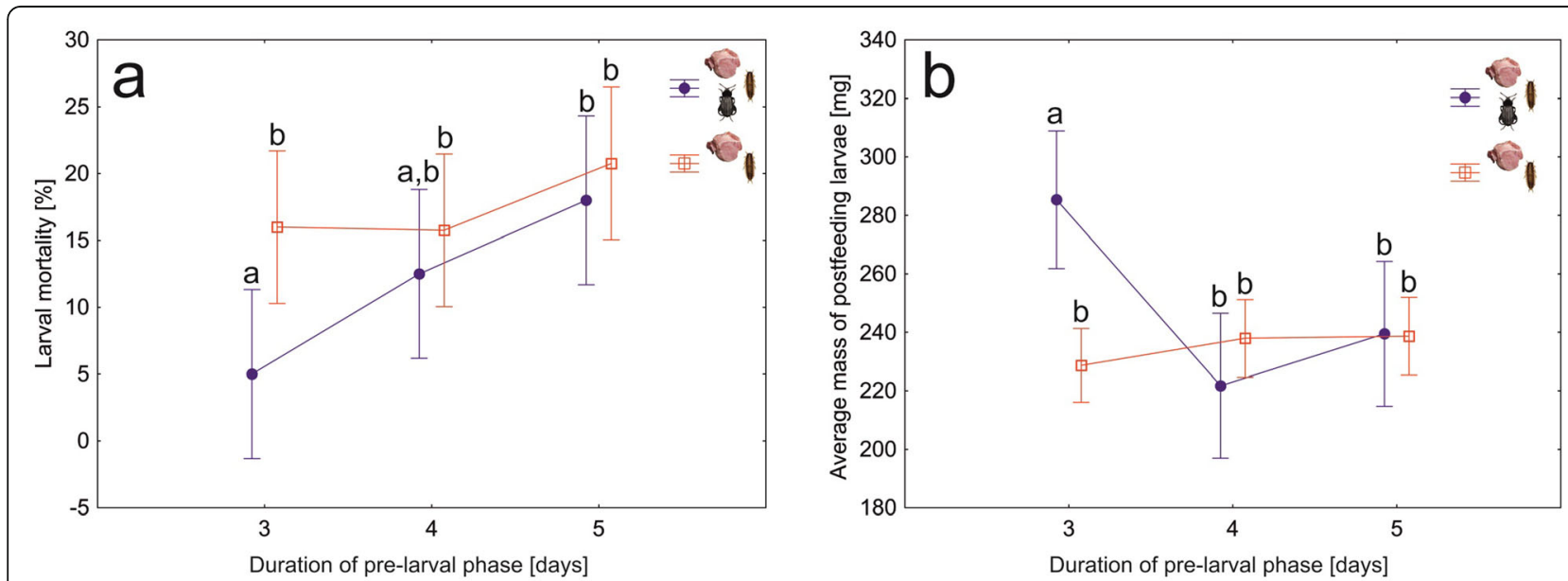

Fig. 7 Larval mortality (a) and average mass of postfeeding larvae (b) for Necrodes littoralis colonies differing in the presence of adult beetles during the pre-larval phase and in the duration of this phase. Symbols - means, whiskers - 95\% confidence intervals, different letters denote significant differences in pairwise comparisons 
directly by the beetles or through carrion digestion [24, 26]. Microbes involved in composting produce heat as a by-product of their metabolic processes [61]. This mechanism is used by megapode birds and crocodilians to incubate their eggs [6-9]. A similar mechanism may be harnessed by Necrodes beetles to produce heat on carrion. Heat may however also be produced through exothermal digestion of carrion by beetle exudates. Exothermic digestion has been hypothesized to produce heat in the aggregations of blow fly larvae, although there is no detailed description for this mechanism or evidence in support [18].

The heat of a matrix may benefit Necrodes larvae in several ways. By increasing larval development rate, heat may reduce time larvae spend on carrion. Our results support this interpretation. When we compared M/A + $\mathrm{L}$ and $\mathrm{M}+\mathrm{L}$ colonies, we found that the heat production in a matrix increased during the larval feeding phase and larval development times became shorter, following the application of exudates by adult beetles $(\mathrm{M} / \mathrm{A}+\mathrm{L}$ colonies). The colonies differed only in the presence of adult beetles and the application of their exudates on meat in the pre-larval phase. Accordingly, heat and development effects recorded in this experiment may be robustly attributed to these differences. Because, similarly to other insects, $N$. littoralis reveals an increase in development rate and a decrease in development time with an increase in a rearing temperature [59], the larger heat of the matrix must have resulted in shorter development time of the larvae. Linking shorter development with an increase in the matrix heat is the most credible and parsimonious interpretation of our results. Exudates of adult burying beetles inhibit carrion decay, facilitate its digestion and change its microbiome [24, 26]. Although these effects have not been reported for Necrodes beetles, it is probable that at least some of them occur in this species, and may enhance development, similarly to the matrix heat. From this point of view, shorter larval development may be an effect of the cumulative action of the matrix heat and other matrix-related factors. Interestingly, shorter larval development times were found also for Nicrophorus mexicanus [62] and Nicrophorus vespilloides [28] in full care or prehatch care conditions (i.e. with exudates application by adult beetles) as compared to the no care conditions, and for Nicrophorus orbicollis [63] in the biparental care conditions (i.e. more exudates) as compared to the uniparental care conditions. Although no data exist on the heat production in the feeding matrix formed by burying beetles, it is tempting to hypothesize that these effects, similarly to Necrodes beetles, resulted from the lagging thermal benefits of carrion preparation by adult Nicrophorus beetles.

Pukowski [25] suggested that smearing carrion with anal exudates may favour aggregation of Nicrophorus larvae. Larvae of $N$. littoralis consistently respond to heat in their environment, they aggregate in the hot spots and respond to changes in the hot spot location by relocating themselves [14]. Accordingly, heat of the matrix may stimulate larvae to form aggregations in a suitable site for their feeding.

However, the benefits of Necrodes larvae may be less obvious. The heat of the matrix may inhibit or support the growth of microbial components of the matrix. Studies of burying beetles linked the microbial suppression on carrion with the production of antimicrobial compounds $[24,26,29,38,39,41]$. The heat of the matrix may act synergistically with antimicrobials. There is evidence, from other insect groups, that heat may act in this way. A wax moth larvae Galleria mellonella (Lepidoptera: Pyralidae), colonizing dead or weak honeybee colonies, were found to elevate temperature when aggregated inside beehives [5, 64]. A recent study showed substantial mortality of Galleria larvae after infection with Metarhizium fungi at $24{ }^{\circ} \mathrm{C}$, whereas at $34^{\circ} \mathrm{C}$ a 10 fold higher dose of the fungus was necessary to reach similar mortality rate, indicating that temperature elevation by communally feeding Galleria larvae suppresses entomopathogenic fungi [65]. Consequently, the larger heat production in the matrix formed by larvae after application of exudates by adult Necrodes beetles, and the lower mortality in such colonies suggest that the matrix heat may facilitate suppression of microbial competitors and favour beetle survival on carrion. Heat of a matrix may also support growth or activity of mutualistic microbes. Duarte et al. [35] hypothesized that Nicrophorus beetles seed their inner microbes on carrion or replant microbes from the carrion gut to its surface. In a similar way blow flies were demonstrated to inoculate carrion with regurgitations or feces containing microbes that in effect facilitate aggregation of flies; the so-called 'fly factor' [66]. From the other side, Miller et al. [67] found little similarity between microbiomes of adult Nicrophorus defodiens gut, their anal secretions and prepared carrion, suggesting that only key microbes are transmitted from the beetle gut to the carcass. Recent analyses of carrion beetle microbiomes recurrently reported an abundant presence of Yarrowia yeasts in the beetle gut and on carrion, which suggests a mutualistic link between the yeasts and the beetles $[24,26,36,42,43]$. Microbial mutualists are probably also associated with Necrodes beetles, and the heat of the matrix may support their growth or activity.

Our hypotheses on the benefits of the matrix heat are not mutually exclusive. Moreover, heat is not the only attribute of the matrix that is beneficial for the beetles. We assume 
that microbial and enzymatic content of the matrix brings clear benefits for Necrodes larvae, similarly to the Nicrophorus larvae. Therefore, our results on the larval mortality most likely resulted from the cumulative action of the heat and the other beneficial attributes of the matrix.

Interestingly, we found that larval mortality increased when we extended the pre-larval phase. The effect was present in $\mathrm{M} / \mathrm{A}+\mathrm{L}$ and $\mathrm{M}+\mathrm{L}$ colonies. It was very clear in $\mathrm{M} / \mathrm{A}+\mathrm{L}$ colonies, and because adult Necrodes beetles were feeding on the meat and we did not supplement it, this must have decreased the quantity of meat available for the larvae and as a consequence their survival. Extension of the pre-larval phase decreases the quality of meat as well, due to the microbial decomposition. Although spreading the exudates of adult beetles probably suppresses putrefaction [37], it can have the inhibitory effect on decomposition mainly on the meat surface and does not inhibit microbes in the inner layers of meat. Application of exudates by adult beetles has a rather quantitative effect that suppresses but not eliminates putrefaction. Thus, the decreasing quantity and quality of meat with the extension of the pre-larval phase could explain these results.

Although scenarios for the evolution of behavioural strategies of spreading the exudates over carrion are highly speculative, several hypotheses may be considered in this respect. Suppression of putrefaction, heat production, facilitating exodigestion and enhancing the growth of beneficial microbes might have exerted selection pressures here. The extent to which these effects were important for different carrion beetles is open to future research, but it will be very difficult to study these effects in isolation. Moreover, their importance certainly differs between the species and also between the life stages of the species. Necrodes beetles are communal breeders, abundantly colonizing large cadavers [57, 68], whereas Nicrophorus beetles usually breed individually, monopolizing small cadavers [12, 25]. Therefore, spreading the exudates may be associated with different constraints and benefits in these beetles.

An increase in heat emission of the matrix formed by larvae after the application of exudates by adult beetles in the pre-larval phase, in association with a decrease of larval development time and mortality, may be regarded as a new form of indirect parental care with an important thermal component. This is also the first demonstration of parental care among Silphinae beetles. Although anal secretions of Necrodes surinamensis were found to have antibacterial action [37], no previous study of Necrodes beetles reported that they spread the exudates over carrion or that spreading the exudates enhances their offspring's fitness. Surprisingly, Hoback et al. indicated in their paper that anal secretion's use "... as a preservative seems unlikely because $N$. surinamensis arrives at large $(>500 \mathrm{~g})$ carcasses and its larvae feed on maggots" [37]. The other studies on anal secretions of Necrodes beetles were focused on their defensive function $[55,56]$. The hypothesis of parental care of Necrodes beetles needs further studies and more data in support, as there are alternative explanations for the behaviour of adult Necrodes beetles on carrion. The beetles may simply feed on carrion while engaging in reproduction and through defecation they may increase a microbial load on carrion surface with side effects for their offspring.

Highly developed parental care occurs in Nicrophorus beetles (Nicrophorinae), with concealment and preparation of small carrion, provisioning of pre-digested food for young larvae and defence of brood or carrion against competitors [12, 25]. Full parental care of Nicrophorus beetles results in higher larval survival and mass, compared to broods without parental care [28, 69]. Simple parental care, in the form of clearing carrion of fly larvae and brood guarding was also described in Ptomascopus beetles (Nicrophorinae) [70, 71]. Our findings suggest that indirect forms of parental care, particularly carrion manipulations bringing deferred benefits for the larvae, may be more common among carrion beetles.

Feeding matrixes formed by other insect groups on various substrates may produce heat, equally to the matrix formed on carrion by Necrodes beetles. Blow fly larvae (Diptera: Calliphoridae) while feeding on carrion elevate the temperature inside their aggregation [18, 72]. Similarly, wax moth larvae Galleria mellonella (Lepidoptera: Pyralidae) elevate the temperature when feeding in aggregation inside a beehive $[5,64]$. Thermogenesis may occur in these cases in the feeding matrix that is formed on carrion or inside bee chambers by the larvae. Although such thermogenesis is not known in other insect groups that form the matrix, e.g. burying beetles [24], our findings suggest that it may be more frequent, particularly among species that feed on rich and bulky resources, e.g. carrion, fallen fruits or dung.

Because thermogenesis of carrion insects (e.g. blow flies or Necrodes beetles) affects their development time, it is also of relevance for the estimation of post-mortem interval (PMI) in forensic entomology. Heat production in larval aggregations has been thoroughly studied in case of blow flies [18, 72]; however, current protocols for PMI estimation do not account for insect-driven thermogenesis. Therefore, understanding the patterns and mechanisms of thermogenesis in carrion insects may have fascinating implications for the PMI estimation based on insect evidence.

\section{Methods}

Beetle colony maintenance and general rearing protocols Laboratory colony was established using adult beetles sampled from pig carcasses in alder forest of Biedrusko military range $\left(52^{\circ} 31^{\prime} \mathrm{N}, 16^{\circ} 54^{\prime} \mathrm{E}\right.$; Western Poland). Beetles 
were kept in rearing containers (20-30 insects per container, sex ratio about 1:1) on humid soil, at room temperature $\left(20-23^{\circ} \mathrm{C}\right)$ and humidity (50-60\%). Three to five containers were usually maintained at the same time in the laboratory. Colonies were provided with fresh pork meat ad libitum (raw pork in pieces: shoulder, neck or ham).

All experiments were performed in rearing containers with a volume of 1.51 (initial experiments and experiment 1) or $3.5 \mathrm{l}$ (experiment 2) with about $5 \mathrm{~cm}$ of humid soil. The soil was not autoclaved. On one side of container meat was placed, and on the opposite side, we put wet cotton wool to maintain high humidity inside and water for the beetles (Fig. 3). To prevent the meat from drying out and to mimic skin that covers carrion and below which $N$. littoralis usually feed and breed, we put aluminium foil over the entire surface.

$N$. littoralis lays eggs (30-50 per batch) to the soil. There are three larval instars. After the third instar larva ceases feeding, it buries itself in the soil, forms a pupal chamber where it pupates. After emergence, adult beetle stays for some time in the chamber, then it digs itself out fully coloured.

\section{Thermal imaging and temperature quantification}

We monitored thermal conditions inside beetle colonies using thermal imaging camera Testo $885-2$ with $30^{\circ} \times$ $23^{\circ}$ infrared lens (Testo, Germany), mounted to a tripod while making images. Images were taken in room temperature and humidity $\left(20-23^{\circ} \mathrm{C}, 50-60 \%\right)$, with rearing containers taken outside of a temperature chamber and images made within no more than $2 \mathrm{~min}$.

Based on initial tests with meat, all temperature measurements were made with the emissivity set at 0.8 and reflected temperature set at $17^{\circ} \mathrm{C}$. Heat production of the matrix-covered surface was defined as a difference between the average temperature of the surface and the background. To obtain background surface temperatures, we measured average temperature in an area of clean soil located in the meat-opposite side of the container. These measurements were averaged for the first three days starting from the colony establishment. During these initial days, the matrix temperature was usually only a little higher than the background temperature, so the heat production in the matrix had negligible effects on the background temperature. Because the matrix enlarged with colony age, and at some time it covered also the soil that surrounded meat, it was impossible to quantify heat production always within the same area. Instead, we quantified the average temperature in the largest possible circular or ellipsoidal area, depending on the shape of the surface covered with the matrix. We used for this purpose in-built tools of the IRSoft 4.5 software (Testo, Germany).

\section{Initial experiments}

To determine the optimal number of larvae and the optimal quantity of meat, we performed several initial trials. By comparing heat production in larval colonies of various abundance (10, 20, 30, 40, 50 and 100 larvae), we found that normal growth and heat production were already present in colonies of 40 larvae (Fig. III in supplementary material). Accordingly, we used such colonies in the main experiments. During initial trials, we also tested setups with different quantity of meat and decided to use $2 \mathrm{~g}$ per larva in Experiment 1 and $3.5 \mathrm{~g}$ per larva in Experiment 2. In Experiment 2 we investigated the effect of heat in a feeding matrix on larval fitness, so we decided to provide larvae with more meat to maintain their optimal growth.

\section{Environmental temperature and heat production in feeding matrix (Experiment 1)}

To test the effect of rearing temperature on the heat production in the matrix, we compared heat emission of the matrix across larval colonies reared under constant temperatures of $16,18,20$ and $23^{\circ} \mathrm{C}$. Heat production in larval colonies $(\mathrm{M}+\mathrm{L}$ setup, 40 larvae; $80-85 \mathrm{~g}$ of raw pork in pieces: shoulder, neck or ham) was compared against heat production in paired containers with meat only ( $\mathrm{M}$ setup, $80-85 \mathrm{~g}$ of pork). Ten pairs of containers (replicates) were studied in each temperature (nine pairs in $20^{\circ} \mathrm{C}$ ). To establish experimental colonies we sampled freshly hatched first instar larvae from our main colony. Containers were kept in temperature chambers (ST 1/1 BASIC or + , POL EKO, Poland). Two temperatures were studied at the same time. Experiments in 18 and $23^{\circ} \mathrm{C}$ started on 28 January 2019 and in 16 and $20^{\circ} \mathrm{C}$ on 19 March 2019. To keep thermal conditions as close as possible within the replicate pairs of containers, we kept each pair on the same shelf in the chamber. Once a day colonies were taken out of a chamber to make thermal images. Results were analysed using ANOVA for repeated measures designs in Statistica 13 (TIBCO Software Inc., US), with rearing temperature as an independent variable, container type $(\mathrm{M}+\mathrm{L}$ or $\mathrm{M}$ setups) in a pair as a repeated measures variable and the average heat production in the matrix (i.e. heat production averaged across measurement days) as a dependent variable. No outliers were detected. Fisher LSD test was used for post-hoc pairwise comparisons.

\section{Heat production and beetle development in different colonies (experiment 2)}

Our main experiment compared thermal conditions and beetle development between two types of colonies in a paired experimental design (Fig. 1). The first colony type had adult beetles in the pre-larval phase and larvae in the larval feeding phase (M/A $+\mathrm{L}$ setup). The second type had no adult beetles in the pre-larval phase and 
larvae in the larval feeding phase ( $\mathrm{M}+\mathrm{L}$ setup). Because we also wanted to test, if the length of the pre-larval phase affects larval fitness, we used three durations of this phase ( 3,4 and 5 days). Each treatment was replicated 10 times. Pairs of colonies $(\mathrm{M} / \mathrm{A}+\mathrm{L}$ and $\mathrm{M}+\mathrm{L}$ setups) were replicates. In each pair, insect colonies were established using larvae hatched at the same time and sampled at random from the same rearing container, meat from the same piece of pork and soil from the same package. Replicate pairs were kept on the same shelf in a temperature chamber at $23^{\circ} \mathrm{C}$ (ST $1 / 1$ BASIC or + , POL EKO, Poland). Beetles were provided with $145-150 \mathrm{~g}$ of pork (raw pork in pieces from shoulder, neck or ham) in both setups. In the $\mathrm{M} / \mathrm{A}+\mathrm{L}$ setup 10 adult beetles (sampled at random from our main colony, sex ratio 1:1) were kept on meat for the duration of 3, 4 or 5 days, depending on the treatment. Afterwards, the meat was transferred to a new container and 40 freshly hatched first instar larvae were added. Meat relocation was necessary because adult beetles usually oviposited during the pre-larval phase and it was difficult to control the number of larvae in the colonies. In the $\mathrm{M}+\mathrm{L}$ setup, meat was decomposing without insects for the duration of 3, 4 or 5 days, depending on the treatment. Then, it was transferred to a new container and 40 freshly hatched first instar larvae were added to the container. Experiments started on 3 March 2019 (3 replicates), 15 March 2019 (7 replicates), 8 May 2019 (10 replicates) and 5 August 2019 (10 replicates). Once a day colonies were taken out of the chambers to make thermal images.

After larvae stopped feeding and began to bury themselves, we counted and weighed them (laboratory scale AS 82/220.R2, Radwag, Poland). Then, larvae were transferred to the soil-filled Petri dishes (3 larvae per dish), where we monitored further development to determine pupation and eclosion times. Results were analysed using ANOVA for repeated measures designs in Statistica 13 (TIBCO Software Inc., US), with the duration of the prelarval phase as an independent variable and a container type $(\mathrm{M} / \mathrm{A}+\mathrm{L}$ or $\mathrm{M}+\mathrm{L}$ setups) in a pair as a repeated measures variable. The average heat production in the pre-larval phase and the larval feeding phase, the average (per colony) larval and pupal development times, larval mortality and the average (per colony) mass of postfeeding larvae were used as dependent variables in separate analyses. No outliers were detected. Fisher LSD test was used for post-hoc pairwise comparisons.

\section{Supplementary Information}

The online version contains supplementary material available at https://doi. org/10.1186/s12983-020-00385-7.

Additional file 1 (supplementary figures).

Additional file 2 (datasets).

\section{Acknowledgments}

The study was funded by the National Science Centre of Poland (grant no. 2016/21/B/NZ8/00788). We would like to thank anonymous reviewers for their comments that helped us to improve the manuscript.

\section{Authors' contributions}

S.M. developed the concept for the study and the article, analysed the data and wrote the manuscript. Both authors performed experiments, prepared raw data for analyses, discussed the results, prepared figures and reviewed the manuscript. Both authors read and approved the final manuscript.

\section{Availability of data and materials}

The datasets supporting this article have been uploaded as part of the supplementary material.

\section{Ethics approval and consent to participate}

This manuscript describes laboratory experiments using carrion beetle species $N$. littoralis. The species is not under protection. No permission or approval from the Ethics Commission was needed. Pig carcasses were used as a bait to sample insects to establish a laboratory colony. The carcasses were bought from the local pig farm. Because the field study consisted of experiments on carcasses only, no approval from ethic commission was needed.

\section{Competing interests}

We have no competing interests to declare.

Received: 1 October 2020 Accepted: 30 December 2020

Published online: 02 February 2021

\section{References}

1. Sanborn A. Thermoregulation in insects. In: Encyclopedia of Entomology. Dordrecht: Springer Netherlands; 2008. p. 3757-60.

2. Heinrich B. Thermoregulation. In: Encyclopedia of Insects: Elsevier; 2009. p. 993-9.

3. Angilletta MJ. Thermal adaptation: a theoretical and empirical synthesis: Oxford University Press; 2009

4. May ML. Insect thermoregulation. Annu Rev Entomol. 1979;24(1):313-49.

5. Heinrich B. The hot-blooded insects: strategies and mechanisms of thermoregulation. Berlin, Heidelberg: Springer Science \& Business Media; 1993.

6. Seymour RS, Ackerman RA. Adaptations to underground nesting in birds and reptiles. Am Zool. 1980;20(2):437-47.

7. Seymour RS, Bradford DF. Temperature regulation in the incubation mounds of the Australian brush-Turkey. Condor. 1992;94(1):134-50.

8. Magnusson WE, Lima AP, Sampaio RM. Sources of heat for nests of Paleosuchus trigonatus and a review of crocodilian nest temperatures. J Herpetol. 1985:199-207.

9. Murray CM, Crother Bl, Doody JS. The evolution of crocodilian nesting ecology and behavior. Ecol Evol. 2020;10(1):131-49.

10. Ruf C, Fiedler K. Thermal gains through collective metabolic heat production in social caterpillars of Eriogaster lanestris. Naturwissenschaften. 2000;87(4):193-6.

11. Payne JA. A summer carrion study of the baby pig Sus scrofa Linnaeus. Ecology. 1965;46(5):592-602.

12. Scott MP. The ecology and behavior of burying beetles. Annu Rev Entomol. 1998;43:595-618.

13. Anderson GS, Barton PS, Archer M, \& Wallace JR (2019) Invertebrate scavenging communities. Carrion Ecology and Management, eds Olea PP, Mateo-Tomás P, \& Sánchez-Zapata JA (Springer International Publishing, (ham), 45-69.

14. Gruszka J, et al. Patterns and mechanisms for larval aggregation in carrion beetle Necrodes littoralis (Coleoptera: Silphidae). Anim Behav. 2020;162:1-10.

15. Girard M. Etudes sur la chaleur libre degagee par les animaux invertebres et specialement les insectes: Victor Masson; 1869

16. Deonier C. Carcass temperatures and their relation to winter blowfly populations and activity in the southwest. J Econ Entomol. 1940;33:166-70.

17. Turner B, Howard T. Metabolic heat generation in dipteran larval aggregations: a consideration for forensic entomology. Med Vet Entomol. 1992;6(2):179-81.

18. Slone $\mathrm{DH}$, Gruner SV. Thermoregulation in larval aggregations of carrionfeeding blow flies (Diptera: Calliphoridae). J Med Entomol. 2007;44(3):516-23. 
19. Charabidze D, Bourel B, Gosset D. Larval-mass effect: characterisation of heat emission by necrophageous blowflies (Diptera: Calliphoridae) larval aggregates. Forensic Sci Int. 2011;211(1-3):61-6.

20. Matuszewski S, Bajerlein D, Konwerski S. Active decay driven by larval Necrodes littoralis L. (Coleoptera: Silphidae). In: 8th Meeting of the European Association for Forensic Entomology. Murcia: Universidad de Murcia; 2010. p. 39.

21. Rodriguez WC 3rd, Bass WM. Decomposition of buried bodies and methods that may aid in their location. J Forensic Sci. 1985;30(3):836-52.

22. Johnson AP, Mikac KM, Wallman JF. Thermogenesis in decomposing carcasses. Forensic Sci Int. 2013;231(1-3):271-7.

23. Rivers DB, Thompson C, Brogan R. Physiological trade-offs of forming maggot masses by necrophagous flies on vertebrate carrion. Bull Entomol Res. 2011;101(5):599-611.

24. Shukla SP, et al. Microbiome-assisted carrion preservation aids larval development in a burying beetle. Proc Natl Acad Sci U S A. 2018:115(44):11274-9.

25. Pukowski E. Ökologische untersuchungen an Necrophorus F. Z Morphol Ökol Tiere. 1933;27(3):518-86.

26. Vogel $\mathrm{H}$, et al. The digestive and defensive basis of carcass utilization by the burying beetle and its microbiota. Nat Commun. 2017:8:15186.

27. Degenkolb T, Düring R-A, Vilcinskas A. Secondary metabolites released by the burying beetle Nicrophorus vespilloides: chemical analyses and possible ecological functions. J Chem Ecol. 2011;37(7):724-35.

28. Rozen DE, Engelmoer DJ, Smiseth PT. Antimicrobial strategies in burying beetles breeding on carrion. Proc Natl Acad Sci U S A. 2008;105(46):17890-5.

29. Arce AN, Johnston P, Smiseth PT, Rozen DE. Mechanisms and fitness effects of antibacterial defences in a carrion beetle. J Evol Biol. 2012;25(5):930-7.

30. Suzuki S. Suppression of fungal development on carcasses by the burying beetle Nicrophorus quadripunctatus (Coleoptera: Silphidae). Entomol Sci. 2001;4(4):403-5.

31. Cotter SC, Kilner RM. Sexual division of antibacterial resource defence in breeding burying beetles, Nicrophorus vespilloides. J Anim Ecol. 2010;79(1): 35-43.

32. Suzuki S. Does carrion-burial by Nicrophorus vespilloides (Silphidae: Coleoptera) prevent discovery by other burying beetles? Entomol Sci. 1999; 2(2):205-8.

33. Trumbo ST, Sikes DS, Philbrick PK. Parental care and competition with microbes in carrion beetles: a study of ecological adaptation. Anim Behav. 2016;118:47-54.

34. Shukla SP, Vogel H, Heckel DG, Vilcinskas A, Kaltenpoth M. Burying beetles regulate the microbiome of carcasses and use it to transmit a core microbiota to their offspring. Mol Ecol. 2018;27(8):1980-91.

35. Duarte A, Welch M, Swannack C, Wagner J, Kilner RM. Strategies for managing rival bacterial communities: lessons from burying beetles. J Anim Ecol. 2018;87(2):414-27.

36. Wang Y, Rozen DE. Gut microbiota colonization and transmission in the burying beetle Nicrophorus vespilloides throughout development. Appl Environ Microbiol. 2017;83(9):e03250-16

37. Hoback WW, Bishop AA, Kroemer J, Scalzitti J, Shaffer JJ. Differences among antimicrobial properties of carrion beetle secretions reflect phylogeny and ecology. J Chem Ecol. 2004;30(4):719-29.

38. Hall $\mathrm{CL}$, et al. Inhibition of microorganisms on a carrion breeding resource: the antimicrobial peptide activity of burying beetle (Coleoptera: Silphidae) oral and anal secretions. Environ Entomol. 2011:40(3):669-78.

39. Arce AN, Smiseth PT, Rozen DE. Antimicrobial secretions and social immunity in larval burying beetles, Nicrophorus vespilloides. Anim Behav. 2013;86(4):741-5.

40. Reavey CE, Beare L, Cotter SC. Parental care influences social immunity in burying beetle larvae. Ecol Entomol. 2014;39(3):395-8.

41. Jacobs CG, et al. Sex, offspring and carcass determine antimicrobial peptide expression in the burying beetle. Sci Rep. 2016;6:25409.

42. Kaltenpoth M, Steiger S. Unearthing carrion beetles' microbiome: characterization of bacterial and fungal hindgut communities across the Silphidae. Mol Ecol. 2014;23(6):1251-67.

43. Wang Y, Rozen DE. Gut microbiota in the burying beetle, Nicrophorus vespilloides, provide colonization resistance against larval bacterial pathogens. Ecol Evol. 2018;8(3):1646-54

44. Sikes D. Carrion beetles (Coleoptera: Silphidae). In: Capinera J, editor Encyclopedia of Entomology: Springer Netherlands; 2008. p. 749-58.

45. Sikes DS. Silphidae Latreille, 1807. In: Beutel RG, Leschen RAB, editors. Handbook of Zoology, Volume IV. Arthropoda: Insecta Part 38: Walter de Gruyter, Berlin and New York; 2005. p. 288-96.
46. Ikeda H, Kagaya T, Kubota K, Abe T. Evolutionary relationships among food habit, loss of flight, and reproductive traits: life-history evolution in the Silphinae (Coleoptera: Silphidae). Evolution. 2008;62(8):2065-79.

47. King JE, Riegler M, Thomas RG, Spooner-Hart RN. Phylogenetic placement of Australian carrion beetles (Coleoptera: Silphidae). Austral Entomol. 2015; 54(4):366-75.

48. Dobler S, Müller JK. Resolving phylogeny at the family level by mitochondrial cytochrome oxidase sequences: phylogeny of carrion beetles (Coleoptera, Silphidae). Mol Phylogenet Evol. 2000;15(3):390-402.

49. Ratcliffe BC. The natural history of Necrodes surinamensis (FABR.)(Coleoptera: Silphidae). T Am Entomol Soc. 1972:98:359-410.

50. Matuszewski S, Konwerski S, Frątczak K, Szafałowicz M. Effect of body mass and clothing on decomposition of pig carcasses. Int J Legal Med. 2014; 128(6):1039-48.

51. Ratcliffe BC. The carrion beetles (Coleoptera: Silphidae) of Nebraska; 1996.

52. Watson E, Carlton C. Succession of forensically significant carrion beetle larvae on large carcasses (Coleoptera: Silphidae). Southeast Nat. 2005;4(2): 335-46.

53. Matuszewski S, Szafałowicz M. Temperature-dependent appearance of forensically useful beetles on carcasses. Forensic Sci Int. 2013;229(1-3):92-9.

54. Matuszewski S, Bajerlein D, Konwerski S, Szpila K. Insect succession and carrion decomposition in selected forests of Central Europe. Part 3: succession of carrion fauna. Forensic Sci Int. 2011;207(1-3):150-63.

55. Eisner T, Meinwald J. Defensive spray mechanism of a silphid beetle (Necrodes surinamensis). Psyche. 1982;89(3-4):357-67.

56. Eisner T, Deyrup M, Jacobs R, Meinwald J. Necrodols: anti-insectan terpenes from defensive secretion of carrion beetle (Necrodes surinamensis). J Chem Ecol. 1986;12(6):1407-15.

57. Matuszewski S, et al. Effect of body mass and clothing on carrion entomofauna. Int J Legal Med. 2016;130(1):221-32.

58. Matuszewski S, Bajerlein D, Konwerski S, Szpila K. Insect succession and carrion decomposition in selected forests of Central Europe. Part 1: pattern and rate of decomposition. Forensic Sci Int. 2010;194(1-3):85-93.

59. Gruszka J, Matuszewski S. Estimation of physiological age at emergence based on traits of the forensically useful adult carrion beetle Necrodes littoralis L. (Silphidae). Forensic Sci Int. 2020;314(110407).

60. Metcalf $J$, et al. Microbial community assembly and metabolic function during mammalian corpse decomposition. Science. 2016;351(6269):158-62.

61. Ryckeboer J, et al. A survey of bacteria and fungi occurring during composting and self-heating processes. Ann Microbiol. 2003;53(4):349-410.

62. Anduaga S, Huerta C. Effect of parental care on the duration of larval development and offspring survival in Nicrophorus mexicanus Matthews (Coleoptera: Silphidae). Coleopts Bull. 2001;55(3):264-71.

63. Trumbo ST. Reproductive benefits and the duration of paternal care in a biparental burying beetle, Necrophorus orbicollis. Behaviour. 1991;117(1):82-105.

64. Buchmann $\mathrm{S}$, Spangler $\mathrm{H}$. Thermoregulation by greater wax moth larvae. Am Bee J. 1991;131:772-3.

65. Kryukov $W$, et al. Fungal infection dynamics in response to temperature in the lepidopteran insect Galleria mellonella. Insect Sci. 2018;25(3):454-66.

66. Uriel $Y$, et al. The fly factor phenomenon is mediated by interkingdom signaling between bacterial symbionts and their blow fly hosts. Insect Sci. 2020;27(2):256-65

67. Miller CJ, Bates ST, Gielda LM, Creighton JC. Examining transmission of gut bacteria to preserved carcass via anal secretions in Nicrophorus defodiens. PLoS One. 2019;14(12):e0225711.

68. Charabidze D, Vincent B, Pasquerault T, Hedouin V. The biology and ecology of Necrodes littoralis, a species of forensic interest in Europe. Int J Legal Med. 2016;130(1):273-80.

69. Eggert A-K, Reinking M, Müller JK. Parental care improves offspring survival and growth in burying beetles. Anim Behav. 1998;55(1):97-107.

70. Trumbo ST, Kon M, Sikes D. The reproductive biology of Ptomascopus morio a brood parasite of Nicrophorus. J Zool. 2001;255(4):543-60.

71. Suzuki S, Nagano M. Resource guarding by Ptomascopus morio: simple parental care in the Nicrophorinae (Coleoptera: Silphidae). Eur J Entomol. 2006;103(1):245

72. Heaton V, Moffatt C, Simmons T. Quantifying the temperature of maggot masses and its relationship to decomposition. J Forensic Sci. 2014;59(3):676-82.

\section{Publisher's Note}

Springer Nature remains neutral with regard to jurisdictional claims in published maps and institutional affiliations. 